\title{
Validación del Inventario de Violençia y Acoso Psicológico en el Trabajo -IVAPT-PANDO- en Tres Ámbitos Laborales Chilenos
}

\section{VALIDATION OF THE VIOLENCE AND BULLYING AT WORK INVENTORY -IVAPT-PANDO- IN THREE CHILEAN FIELDS OF WORK}

Ximena Díaz Berr' ${ }^{1}$ Amalia Mauro Cardarelli², Juan Pablo Toro Cifuentes ${ }^{3}$, Cristián Villarroel Poblete ${ }^{4}$, Daniela Campos Schwarze ${ }^{5}$

1. Centro de Estudios de la Mujer, Santiago Chile.

2. Centro de Estudios de la Mujer, Santiago, Chile.

3. Programa de Estudios Psicosociales del Trabajo, Universidad Diego Portales, Santiago, Chile.

4. Departamento Salud Ocupacional, Ministerio de Salud, Santiago, Chile.

5. Asociación Chilena de Seguridad, Santiago, Chile.

\section{RESUMEN}

Introducción: En el contexto de las políticas públicas sobre riesgos psicosociales en Chile, se plantea la necesidad de incluir entre ellos la violencia y el acoso psicológico y disponer de instrumentos válidos y confiables para su medida. Objetivos: se propone validar el Inventario de Violencia y Acoso Psicológico en el Trabajo (IVAPT-Pando) en la población laboral chilena. Método: El proceso consistió en: a) validación de contenidos; b) adaptación semántica del instrumento e incorporación de nuevos ítems orientados a medir violencia ejercida por actores externos a la organización; c) aplicación de la versión final del instrumento a una muestra de 700 trabajadores/as para evaluar sus propiedades psicométricas; d) análisis de validez convergente con el GHQ; y, e) elaboración de baremos referenciales. Resultados: Se confirma la presencia de tres factores componentes del instrumento original y la emergencia de un cuarto factor relativo a violencia externa. La consistencia interna y confiabilidad de ambas versiones son estadísticamente significativas. Se confirma la capacidad del instrumento para discriminar la existencia de presencia e intensidad de la violencia psicológica y de acoso laboral en los sectores estudiados. Se presentan correlaciones significativas con la presencia de psicopatología medida a través del GHQ. Conclusiones: El instrumento tiene las propiedades para discriminar y medir la violencia laboral en los sectores estudiados, pero debe atenderse a algunas dificultades en su aplicación.

(Díaz X, Mauro A, Toro J, Villarroel C, Campos D, 2015. Validación del Inventario de Violencia y Acoso Psicológico en el Trabajo -IVAPTPANDO- en Tres Ámbitos Laborales Chilenos. Cienc Trab. Ene-Abr; 17 [52]: 7-14).

Palabras clave: VIOLENCIA EN EL TRABAJO, ACOSO LABORAL, VALIDACIÓN.

\section{ABSTRACT}

Introduction: In the context of public policies on psychosocial risks in Chile, the need arises to include amongst these violence and bullying at work and obtain valid and reliable instruments for its measurement. Objectives: It is proposed to validate the inventory of Violence and Bullying at Work (IVAPT - Pando) in Chilean workforce; Method: The process consisted of: a) content validation; b) semantic adaptation of the questionnaire and its complementarity with the addition of items designed to measure violence by actors external to the organization; c) final application to a total sample of 700 subjects to analyze the psychometric properties of the instrument; d) elaboration of reference scales; and, e) study of convergent validity with the GHQ. Results: The presence of three components of the original instrument is confirmed, and in the modified instrument emerges a fourth factor on violence by actors outside the organization. Internal consistency and reliability of both versions remain high. The instrument's capacity to discriminate the presence and intensity of violence and harassment in the sectors studied is confirmed. Significant correlations are presented with the presence of psychopathology as measured by the GHQ. Conclusions: The instrument is useful for measuring workplace violence but it should be paid attention to some difficulties in its application.

Key words: WORKPLACE VIOLENCE, WORK HARASSMENT, VALIDATION.

\section{INTRODUCCIÓN}

En Chile, como en otros países del mundo, los problemas de salud mental representan una de las primeras causas de morbilidad de la población, lo cual tiende a acrecentarse en los últimos años. Entre las condiciones de trabajo que determinan un mayor riesgo de desarrollar un trastorno mental de origen laboral, se han identificado algunos factores de riesgo psicosocial asociados a las nuevas formas de organización del trabajo que, en el largo plazo, pueden causar serios daños en la salud psicológica de la población trabajadora y que pueden transitar a cuadros de patología mental. ${ }^{1}$ 
No obstante, se ha reconocido internacionalmente que uno de los estresores sociales más importantes de la vida laboral actual es la violencia y el acoso psicológico en el trabajo, reconocidos ambos como uno de los problemas de salud pública que mayor preocupación provocan en el mundo por su creciente magnitud. ${ }^{2-5}$

El año 2011 el Ministerio de Salud, a través del Departamento de Salud Ocupacional, desarrolló un protocolo para la vigilancia de riesgos psicosociales en los lugares de trabajo. Dicho protocolo adoptó el cuestionario SUSESO-ISTAS 21 como instrumento de vigilancia de dichos riesgos, por ser el único cuestionario que se encuentra validado para nuestra realidad. A pesar de los avances que esta regulación garantiza, tanto por la instalación de la temática como por la posibilidad de generar evidencia epidemiológica, este instrumento no consideró la dimensión violencia laboral y acoso psicológico.

A partir de esta situación surge la necesidad de disponer de un instrumento que permita mediciones válidas y confiables de la violencia y el acoso psicológico en el trabajo en Chile, ampliando de ese modo el campo de dimensiones incluidas en el protocolo de vigilancia de riesgos psicosociales adoptado en Chile.

Para responder a esta necesidad, se propuso validar y estandarizar para la población trabajadora chilena el Inventario de Violencia y Acoso Psicológico en el Trabajo (IVAPT-PANDO) diseñado por el Dr. Manuel Pando en México, a fin de generar diagnósticos más certeros de la presencia e intensidad de la violencia y acoso psicológico en los lugares de trabajo. ${ }^{6}$

El inventario se construyó a partir de un listado inicial de 94 ítems que, tras una revisión y validación por parte de expertos de España, Chile, Honduras, México, Perú y Venezuela se redujo a 47. Posteriormente, luego de una aplicación a 234 trabajadores de 14 estados de la República mexicana en 17 giros empresariales diferentes, se obtuvo una versión final de 22 reactivos con una confiabilidad alfa de Cronbach de 0,91.6,7 El instrumento definitivo fue validado y adaptado en España y en algunos países de América Latina (México, Venezuela, Ecuador, Bolivia, Cuba y Colombia). En Chile se han realizado dos estudios exploratorios en muestras pequeñas para validar el test IVAPT-PANDO. Uno, por Macía ${ }^{8}$, aplicado a una muestra piloto de 48 trabajadores de dos centros de salud de atención primaria en la Región Metropolitana. Otro, realizado por González, Pincheira y Moyano ${ }^{9}$, en que aplicaron el instrumento a una muestra de 150 funcionarios pertenecientes a instituciones de orden y seguridad. En ambos casos, sus autores recomiendan nuevos procesos de adaptación y de validación a distintas poblaciones y contextos.

\section{MÉTODO}

\section{Instrumento}

La versión del Inventario sometido a validación en este estudio se compone de 22 reactivos que plantean distintas situaciones o comportamientos constituyentes de acoso y cada una de ellos requiere de 2 respuestas. La primera (columna A) se refiere a la frecuencia en que dicha situación le ha ocurrido a la persona en los últimos 6 meses en su actual trabajo, proporcionando cinco opciones de respuesta (de $0=$ nunca a $4=$ muy frecuentemente). Esta columna indica la presencia e intensidad de conductas que se consideran violencia psicológica.

La segunda respuesta (Columna B) se refiere a si dicha situación le ha ocurrido a la persona con mayor o menor frecuencia que a sus compañeros/as de trabajo. Esto permite determinar la percepción de acoso psicológico, es decir, si existe intencionalidad de dañar a una persona o grupo en particular o si se trata de prácticas organizacionales habituales. En esta columna se ofrecen 3 alternativas de respuesta: "menos que a mis compañeros(as)"; o "igual que a mis compañeros(as)" o "más que al resto de mis compañeros(as)". Tras completar el cuestionario se solicita señalar quiénes son los o las causantes de las situaciones señaladas en los reactivos (superiores, compañeros o subordinados), lo que permite determinar si el acoso es ascendente, horizontal o descendente.

Como etapas previas al proceso de validación y estandarización que se reporta en este estudio, se llevó a cabo una validación de contenidos del instrumento, una adaptación semántica y la incorporación de nuevos ítems orientados a medir violencia ejercida por actores externos a la organización.

a) Validación de contenido

La validación de contenido se realizó a través de una consulta a un panel de expertos/as o de jueces de distintas instituciones públicas y privadas, universidades, administradoras del seguro de la ley 16.744 y representantes sindicales. El instrumento sometido a consulta del panel de expertos/as fue una versión alternativa elaborada por el equipo en la que se introdujeron dos modificaciones importantes: la delimitación del periodo de ocurrencia del comportamiento indicado (6 meses) y la introducción de 5 nuevos reactivos referidos a acoso sexual. El Panel de expertos aceptó la propuesta de delimitación de tiempo, sugirió dejar solo un reactivo referido a acoso sexual y agregar 3 nuevos reactivos con la finalidad de captar la existencia o no de acoso psicológico provocado por personas ajenas a la empresa o institución (clientes, pacientes, apoderados, alumnos), reactivos no incluidos en el instrumento original.

b) Adaptación semántica

La adaptación semántica consistió en la aplicación del instrumento resultante de la etapa anterior a una muestra intencionada de trabajadores y trabajadoras del Retail, Enseñanza y Salud, para lo cual se realizaron 4 talleres, a los que asistieron 19 mujeres y 13 hombres. Como resultado de este proceso se realizó una serie de modificaciones al instrumento referidas a la redacción y lenguaje de los ítems, y a su configuración (formato, claridad en las instrucciones, insistencia en el anonimato y confidencialidad de los datos, entre las principales). El cuestionario final quedó compuesto por 3 secciones: A) el instrumento IVAPT-PANDO con sus 22 reactivos originales, 1 reactivo relativo a acoso sexual, 3 reactivos relativos a violencia externa; $\mathrm{B}$ ) el instrumento GHQ (con sus 12 preguntas) incluido para un estudio de validez convergente; y, C) información sociol-aboral de los/as trabajadores/as de los sectores productivos consultados.

c) Aplicación del instrumento modificado

Evaluación de sus propiedades psicométricas. Se procedió a un análisis factorial de componentes principales, análisis de consistencia interna utilizando el Alpha de Cronbach y análisis de validez convergente usando como criterio el GHQ-12.

Para el análisis de los datos se utilizó el programa estadístico SPSS en su versión 20 para Windows.

\section{Muestra}

La población objetivo fueron los/as trabajadores/as asalariados/as de empresas adheridas a la ACHS pertenecientes a tres sectores de la economía: comercio, salud y educación de tres regiones representativas del volumen de trabajadores del país: 1) Región Metropolitana; 2) Región de Valparaíso; y, 3) Región del BíoBío. Las cuotas muestrales para cada región se establecieron en base a la población ocupada a nivel nacional en cada rama seleccionada 
en cada región. Inicialmente, se estimó un tamaño muestral de 385 trabajadores/as con un 95\% de confianza. Dado que el propósito de este proyecto fue generar evidencia sobre temáticas escasamente medidas en Chile, se optó por sobrerrepresentar la muestra en un 50\% en cada región y rama.

La versión final del instrumento fue aplicada a una muestra no probabilística de 700 trabajadores y trabajadoras de diferentes empresas e instituciones pertenecientes a los sectores señalados de las tres capitales regionales -Gran Santiago, Valparaíso y Concepción-y se focalizó en grupos ocupacionales señalados por la literatura internacional y nacional como los más expuestos a violencia. En el Comercio, en vendedores/as; en Salud, en trabajadores no profesionales y profesionales no médicos; en Educación, en docentes de básica y media. La muestra sobrepasó el tamaño estimado debido a que en algunos sectores se aplicaron más encuestas respondiendo a demandas de los/as trabajadores/as. Esta quedó constituida por un 26\% de hombres y 74\% de mujeres, dada la alta feminización de la fuerza de trabajo de los Sectores de Educación y Salud. El rango de edad del grupo fluctuó entre 18 y 74 años en los hombres y entre 18 y 68 años en las mujeres. El promedio de edad de hombres y mujeres es de 39,8 años (Tabla 1). Los trabajadores que formaron parte del estudio fueron debidamente

Tabla 1.

Distribución de la muestra por sexo y sector productivo.

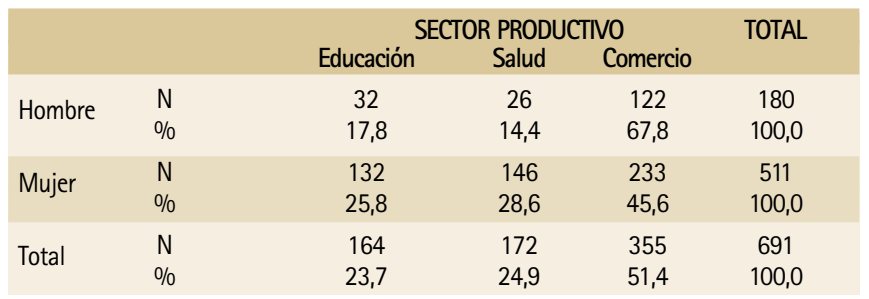

Tabla 2.

Cargas factoriales instrumento original.

\begin{tabular}{|c|c|c|c|c|c|c|c|}
\hline \multicolumn{2}{|c|}{ Componente } & \multicolumn{2}{|c|}{ Eigenvalor inicial } & \multicolumn{2}{|c|}{$\begin{array}{l}\text { Sumas } \\
\text { de extracción de } \\
\text { Cargas Cuadradas }\end{array}$} & \multicolumn{2}{|c|}{$\begin{array}{c}\text { Sumas } \\
\text { de Rotación de } \\
\text { Cargas Cuadradas }\end{array}$} \\
\hline & Total & $\begin{array}{c}\% \text { de } \\
\text { Varianza }\end{array}$ & $\begin{array}{c}\text { Acumulativo } \\
\%\end{array}$ & Total & $\begin{array}{c}\% \text { de } \\
\text { Varianza }\end{array}$ & $\begin{array}{c}\text { Acumulativo } \\
\%\end{array}$ & Total \\
\hline 1 & 11,364 & 51,653 & 51,653 & 11,364 & 51,653 & 51,653 & 10,318 \\
\hline 2 & 1,279 & 5,813 & 57,466 & 1,279 & 5,813 & 57,466 & 8,051 \\
\hline 3 & 1,155 & 5,252 & 62,718 & 1,155 & 5,252 & 62,718 & 7,648 \\
\hline 4 & 0,876 & 3,983 & 66,701 & & & & \\
\hline 5 & 0,844 & 3,836 & 70,538 & & & & \\
\hline 6 & 0,747 & 3,393 & 73,931 & & & & \\
\hline 7 & 0,608 & 2,763 & 76,694 & & & & \\
\hline 8 & 0,542 & 2,465 & 79,160 & & & & \\
\hline 9 & 0,483 & 2,196 & 81,356 & & & & \\
\hline 10 & 0,462 & 2,102 & 83,458 & & & & \\
\hline 11 & 0,438 & 1,991 & 85,448 & & & & \\
\hline 12 & 0,410 & 1,865 & 87,313 & & & & \\
\hline 13 & 0,389 & 1,767 & 89,080 & & & & \\
\hline 14 & 0,350 & 1,591 & 90,671 & & & & \\
\hline 15 & 0,333 & 1,512 & 92,183 & & & & \\
\hline 16 & 0,330 & 1,499 & 93,681 & & & & \\
\hline 17 & 0,312 & 1,417 & 95,098 & & & & \\
\hline 18 & 0,283 & 1,288 & 96,386 & & & & \\
\hline 19 & 0,213 & 0,968 & 97,355 & & & & \\
\hline 20 & 0,207 & 0,941 & 98,296 & & & & \\
\hline 21 & 0,199 & 0,904 & 99,200 & & & & \\
\hline 22 & 0,176 & 0,800 & 100,000 & & & & \\
\hline
\end{tabular}

Método de extracción: Análisis de Componentes Principales. a: Cuando se correlacionan componentes, las sumas de cargas cuadradas no se pueden añadir para obtener una varianza total. informados de los objetivos de la investigación y su participación voluntaria quedó consignada mediante una carta de consentimiento donde se garantiza el anonimato y confidencialidad de los datos entregados por cada uno de los(as) participantes.

\section{RESULTADOS}

\section{Análisis factorial}

Luego de varias pruebas de distintos métodos de rotación o factorización, el equipo de investigación decidió utilizar como método de extracción el análisis por componentes principales, método utilizado por el autor y que se reporta en toda la literatura de validación del instrumento. Este método forma una combinación lineal de las variables observadas. El primer componente es la combinación que da cuenta de la mayor cantidad de varianza explicada. El segundo componente responde a la siguiente cantidad de varianza inmediatamente inferior a la primera y no está correlacionado con el primero. Así, sucesivamente, los componentes explican proporciones menores de la varianza de la muestra total.

Tabla 3.

Estructura matricial instrumento original.

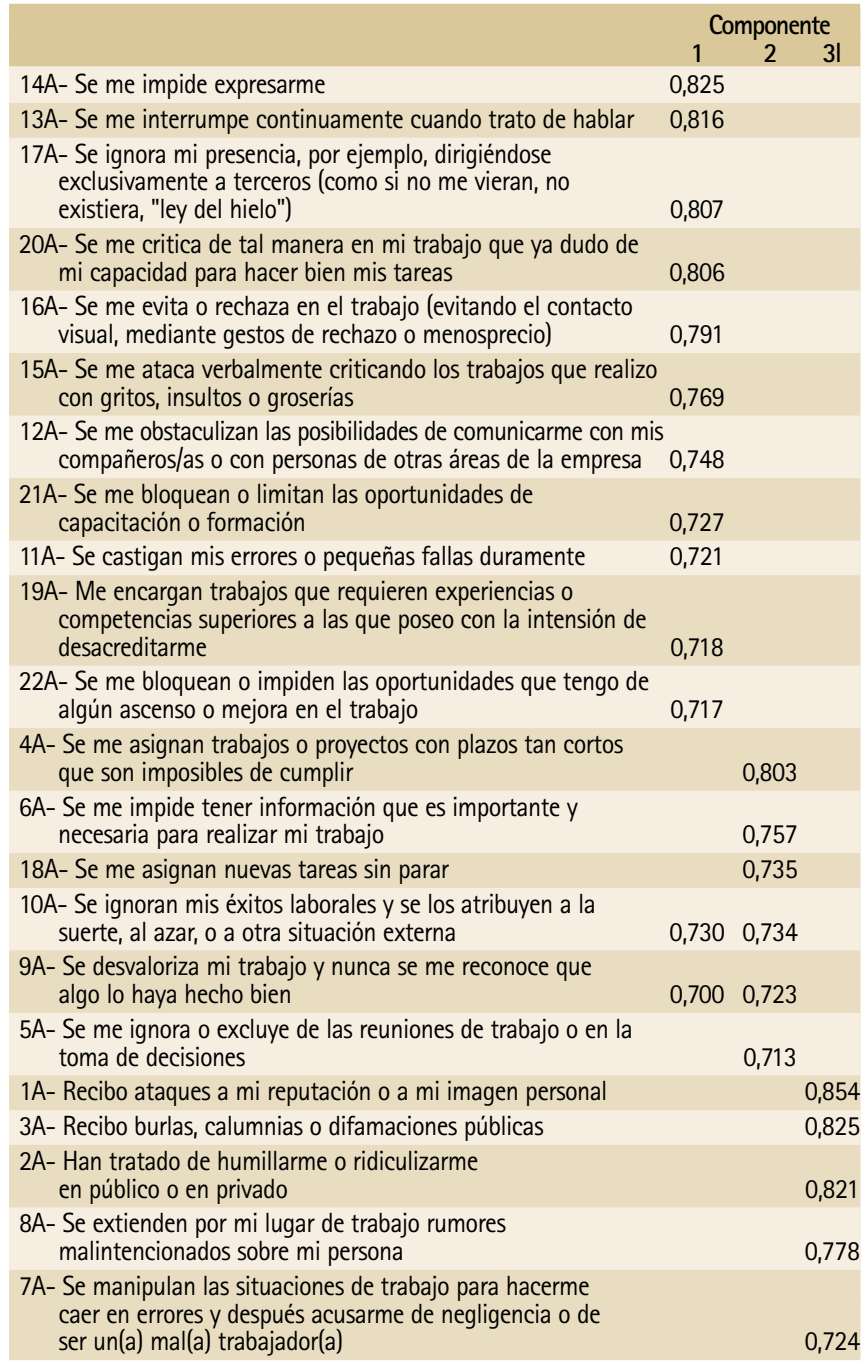


Tabla 4.

Cargas factoriales instrumento modificado.

\begin{tabular}{|c|c|c|c|c|c|c|c|}
\hline \multicolumn{2}{|c|}{ Componente } & \multicolumn{2}{|c|}{ Eigenvalor inicial } & \multicolumn{2}{|c|}{$\begin{array}{l}\text { Sumas } \\
\text { de Extracción de } \\
\text { Cargas Cuadradas }\end{array}$} & \multirow{2}{*}{\multicolumn{2}{|c|}{$\begin{array}{l}\text { Sumas } \\
\text { de Rotación de } \\
\text { Cargas Cuadradas } \\
\text { Acumulativo }\end{array}$}} \\
\hline & Total & $\begin{array}{c}\% \text { de } \\
\text { Varianza }\end{array}$ & $\begin{array}{c}\text { Acumulativo } \\
\%\end{array}$ & & $\begin{array}{c}\% \text { de } \\
\text { Varianza }\end{array}$ & & \\
\hline 1 & 11,874 & 47,497 & 47,497 & 11,874 & 47,497 & 47,497 & 10,665 \\
\hline 2 & 1,658 & 6,633 & 54,130 & 1,658 & 6,633 & 54,130 & 8,332 \\
\hline 3 & 1,281 & 5,123 & 59,253 & 1,281 & 5,123 & 59,253 & 7,947 \\
\hline 4 & 1,139 & 4,557 & 63,810 & 1,139 & 4,557 & 63,810 & 3,978 \\
\hline 5 & 0,884 & 3,538 & 67,347 & & & & \\
\hline 6 & 0,841 & 3,365 & 70,713 & & & & \\
\hline 7 & 0,775 & 3,102 & 73,814 & & & & \\
\hline 8 & 0,656 & 2,623 & 76,437 & & & & \\
\hline 9 & 0,586 & 2,343 & 78,780 & & & & \\
\hline 10 & 0,524 & 2,098 & 80,877 & & & & \\
\hline 11 & 0,466 & 1,863 & 82,740 & & & & \\
\hline 12 & 0,463 & 1,851 & 84,591 & & & & \\
\hline 13 & 0,439 & 1,755 & 86,346 & & & & \\
\hline 14 & 0,405 & 1,620 & 87,966 & & & & \\
\hline 15 & 0,387 & 1,549 & 89,515 & & & & \\
\hline 16 & 0,344 & 1,378 & 90,892 & & & & \\
\hline 17 & 0,333 & 1,332 & 92,224 & & & & \\
\hline 18 & 0,319 & 1,276 & 93,500 & & & & \\
\hline 19 & 0,312 & 1,246 & 94,746 & & & & \\
\hline 20 & 0,292 & 1,166 & 95,912 & & & & \\
\hline 21 & 0,246 & 0,983 & 96,896 & & & & \\
\hline 22 & 0,209 & 0,835 & 97,731 & & & & \\
\hline 23 & 0,198 & 0,790 & 98,522 & & & & \\
\hline 24 & 0,195 & 0,780 & 99,301 & & & & \\
\hline 25 & 0,175 & 0,699 & 100,000 & & & & \\
\hline
\end{tabular}

Método de extracción: Análisis de componentes principales. a: Cuando los componentes se correlacionan, la suma de las cargas cuadradas no se puede agregar para obtener una varianza total.

Respecto al método de rotación, si bien el más utilizado ha sido Varimax, para esta validación se utilizó el método de rotación oblicuo al considerar que los factores están relacionados entre sí, aspecto común dentro de los estudios en ciencias sociales. ${ }^{10}$ Se utilizó un valor eigen mayor a 1 como criterio para determinar el número de factores. Una solución de tres factores fue arrojada tras este. Siguiendo a Matsunaga ${ }^{10}$, se tomó un criterio conservador de .7 para determinar la inclusión de ítems en cada dimensión, por lo que valores menores al criterio fueron suprimidos. Cabe destacar que los criterios de inclusión de esta validación fueron más exigentes que el de otras investigaciones que validaron este instrumento. ${ }^{6-11}$

\subsection{Análisis factorial del instrumento original -22 items}

El análisis factorial se realizó sobre los items de la columna A. Con el fin de revisar la viabilidad del análisis factorial, se aplicó previamente el índice de adecuación muestral de Kaiser-Meyer-Oklin (KMO y el valor de esfericidad de Bartlett), cuyos resultados respaldaron el procedimiento. Los resultados muestran tres componentes que explican un $62,718 \%$ de la varianza. El primer componente explica un 51,653\%, el segundo un 5,813\%, y el tercero un 5,252\%. (Tabla 2).

En cuanto a la estructura matricial, los resultados muestran que 11 items cargan al primer factor, 6 al segundo y 5 al tercero. Dos ítems presentaron cargas cruzadas, 10A y 9A, los cuales fueron asignados a la dimensión con mayor carga (dimensión 2), la que presentó también una mayor coherencia teórica. (Tabla 3).

La revisión del contenido de los items que componen cada uno de los factores identificados nos llevó a proponer la siguiente denominación para cada uno de ellos: Conductas destinadas a desacreditar las competencias laborales del trabajador/a (Factor 1); Intensificación del trabajo, aislamiento y despersonalización de logros (Factor 2); Estigmatización y daño a la imagen pública del trabajador/a (Factor 3).
Tabla 5.

Estructura matricial instrumento modificado.

\begin{tabular}{|c|c|c|c|}
\hline & \multicolumn{3}{|c|}{ Componente } \\
\hline & 1 & 3 & 4 \\
\hline 14A- Se me impide expresarme & 0,826 & & \\
\hline 13A-Se me interrumpe continuamente cuando trato de hablar & 0,814 & & \\
\hline $\begin{array}{l}\text { 20A-Se me critica de tal manera en mi trabajo que ya dudo de } \\
\text { mi capacidad para hacer bien mis tareas }\end{array}$ & 0,809 & & \\
\hline $\begin{array}{l}\text { 17A- Se ignora mi presencia, por ejemplo, dirigiéndose } \\
\text { exclusivamente a terceros (como si no me vieran, } \\
\text { no existiera, "ley del hielo") }\end{array}$ & 0,806 & & \\
\hline $\begin{array}{l}\text { 16A- Se me evita o rechaza en el trabajo (evitando el contacto } \\
\text { visual, mediante gestos de rechazo o menosprecio) }\end{array}$ & 0,797 & & \\
\hline $\begin{array}{l}\text { 15A- Se me ataca verbalmente criticando los trabajos que } \\
\text { realizo con gritos, insultos o groserías }\end{array}$ & 0,770 & & \\
\hline $\begin{array}{l}\text { 12A- Se me obstaculizan las posibilidades de comunicarme } \\
\text { con mis compañeros/as o con personas de otras } \\
\text { áreas de la empresa }\end{array}$ & 0,749 & & \\
\hline $\begin{array}{l}\text { 21A- Se me bloquean o limitan las oportunidades de } \\
\text { capacitación o formación }\end{array}$ & 0,729 & & \\
\hline
\end{tabular}

19A- Me encargan trabajos que requieren experiencias o competencias superiores a las que poseo con la intensión de desacreditarme

22A- Se me bloquean o impiden las oportunidades que tengo de algún ascenso o mejora en el trabajo

$11 \mathrm{~A}$ - Se castigan mis errores o pequeñas fallas duramente

$4 \mathrm{~A}$ - Se me asignan trabajos o proyectos con plazos tan cortos que son imposibles de cumplir

$10 \mathrm{~A}-\mathrm{Se}$ ignoran mis éxitos laborales y se los atribuyen a la suerte, al azar, o a otra situación externa

$6 \mathrm{~A}$ - Se me impide tener información que es importante y necesaria para realizar mi trabajo

9A- Se desvaloriza mi trabajo y nunca se me reconoce que algo lo haya hecho bien

$18 \mathrm{~A}$ - Se me asignan nuevas tareas sin parar

$5 \mathrm{~A}$ - Se me ignora o excluye de las reuniones de trabajo 0 en la toma de decisiones

1A- Recibo ataques a mi reputación o a mi imagen personal

3A- Recibo burlas, calumnias o difamaciones públicas

$2 \mathrm{~A}$ - Han tratado de humillarme o ridiculizarme en público o en privado

$8 \mathrm{~A}$ - Se extienden por mi lugar de trabajo rumores malintencionados sobre mi persona

0,716

0,790

$0,7210,756$

0,751

0,739

0,728

0,711

0,851

0,826

0,821

0,779

A- Se manipulan las situaciones de trabajo para hacerme caer en errores y después acusarme de negligencia o de ser un(a) mal(a) trabajador(a)

25A- He sido objeto de maltrato, humillación, ridiculización, menosprecio, por alguna persona ajena al trabajo como clientes, apoderados, pacientes, estudiantes, etc,

23A- He sido agredido(a) verbalmente por personas ajenas a mi empresa u organización, como clientes, apoderados, pacientes, estudiantes, etc,

24A- He sido agredido(a) fisicamente por personas ajenas a mi empresa u organización como clientes, apoderados, pacientes, estudiantes, etc.

Método de extracción: Análisis de los componentes principales.

\subsection{Análisis factorial incluyendo nuevos items}

Se excluyó del análisis factorial el ítem 23 referido a acoso sexual por cuanto, a diferencia de todos los otros items que apuntan a situaciones propias, este apunta a la percepción de una situación externa. $\mathrm{Al}$ igual que lo realizado en el análisis factorial del instrumento IVAPT-PAND0 original de 22 items, en este caso se utilizó también como método de extracción el análisis por componentes principales y un valor eigen mayor a 1 como criterio para determinar el número de factores. Una solución de cuatro factores fue arrojada tras este análisis. Se tomó un criterio conservador de 7 para determinar la inclusión de items en cada dimensión, por lo que valores menores al criterio fueron suprimidos.

Al igual que en el análisis anterior, la prueba de adecuación muestral de Kaiser-Meyer-Olkin y la prueba de esfericidad de Bartlett indican 
que es viable realizar un análisis factorial en los datos presentados. En cuanto a las comunalidades, no se encuentran valores bajos, por lo que no se excluye ningún ítem bajo este criterio (todos los valores sobre .5). Esto quiere decir que las variables quedan explicadas a través de los componentes extraídos.

Se obtienen cuatro componentes, que explican el 63,810\% de la varianza. El primer componente explica el 47,497\% de la varianza, el segundo el 6,633\%, el tercero el 5,123\% y, el cuarto, constituido por los items agregados por este estudio, el 4,557\%. (Tabla 4).

En cuanto a la estructura matricial del instrumento modificado, comparando esta versión con la original de 22 reactivos, se mantienen los mismos items en cada componente, y aquellos que indican violencia por parte de una persona externa -clientes, apoderados, pacientes, estudiantes, etc.- forman un nuevo factor. A diferencia de lo observado en el instrumento original, esta vez sólo un item presenta carga cruzada (10A). (Tabla 5).

El instrumento modificado quedaría de la siguiente forma incluyendo este nuevo factor:

Conductas destinadas a desacreditar las competencias laborales del trabajador/a (Factor 1); Intensificación del trabajo, aislamiento y despersonalización de logros (Factor 2); Estigmatización y daño a la imagen pública del trabajador/a (Factor 3); Conductas de violencia generadas por personas externas a la empresa u organización (Factor 4).

\section{Análisis de confiabilidad - consistencia interna}

\subsection{Confiabilidad escala original}

La escala total presenta un buen índice de confiabilidad con un alpha de Cronbach con valores que oscilan entre 0,951 y 0,953. (Tabla 6).

\subsection{Confiabilidad escala modificada}

En conclusión, la escala total presenta una confiabilidad de nivel excelente con un alpha de 0,951. La confiabilidad de las subescalas oscila entre un alpha de 0,773 a 0,930, presentando buenos índices de confiabilidad en general. Se recomienda mantener todos los items debido a los argumentos expuestos con anterioridad. De acuerdo a los datos, los ítems agregados constituyen un nuevo factor que, a pesar de que alude a conductas y situaciones que no forman parte de las condiciones internas de trabajo, ni son promovidas por los compañeros o superiores, sí forman parte de las condiciones generales de trabajo y permiten configurar un cuadro más completo de la exposición a la violencia en el contexto laboral. Estimamos, por lo tanto, que estos reactivos arrojan información relevante y pueden formar parte de este instrumento.

\section{Análisis de convergencia. Correlaciones entre IVAPT PANDO y GHQ-12}

Se presentan correlaciones significativas entre ambas versiones del IVAT- Pando y el GHQ-12. Estas correlaciones son levemente más intensas en el instrumento, que incluye los nuevos items relativos a violencia ejercida por terceros externos a la organización. Todas las correlaciones se mantienen como significativas. (Tablas 8 y 9).

\section{Obtención de baremos referenciales}

La aplicación del instrumento a la muestra de 700 trabajadores/as permite extraer baremos interpretativos de los puntajes obtenidos en las escalas componentes. Las categorias construidas varian según se

Tabla 6.

Correlación item - escala total (intrumento original, 22 ítems).

\begin{tabular}{|c|c|c|c|c|}
\hline & $\begin{array}{c}\text { Media de } \\
\text { la Escala si el } \\
\text { ítem se elimina }\end{array}$ & $\begin{array}{l}\text { Varianza de } \\
\text { escala si el } \\
\text { ítem se elimina }\end{array}$ & $\begin{array}{l}\text { İtem } \\
\text { correlacionado- } \\
\text { correlación total }\end{array}$ & $\begin{array}{l}\text { Alfa de Cronbach } \\
\text { si el ítem } \\
\text { se elimina }\end{array}$ \\
\hline 1A- Recibo ataques a mi reputación o a mi imagen personal & 21,13 & 299,935 & 0,632 & 0,952 \\
\hline 2A- Han tratado de humillarme o ridiculizarme en público o en privado & 21,15 & 298,453 & 0,658 & 0,952 \\
\hline 3A- Recibo burlas, calumnias o difamaciones públicas & 21,32 & 299,356 & 0,656 & 0,952 \\
\hline 4A- Se me asignan trabajos o proyectos con plazos tan cortos que son imposibles de cumplir & 20,84 & 299,389 & 0,567 & 0,953 \\
\hline 5A-Se me ignora o excluye de las reuniones de trabajo o en la toma de decisiones & 20,93 & 298,024 & 0,578 & 0,953 \\
\hline 6A- Se me impide tener información que es importante y necesaria para realizar mi trabajo & 21,09 & 297,554 & 0,651 & 0,952 \\
\hline $\begin{array}{l}\text { 7A- Se manipulan las situaciones de trabajo para hacerme caer en errores y después acusarme de } \\
\text { negligencia o de ser un(a) mal(a) trabajador(a) }\end{array}$ & 21,36 & 297,277 & 0,722 & 0,951 \\
\hline $8 \mathrm{~A}$ - Se extienden por mi lugar de trabajo rumores malintencionados sobre mi persona & 20,99 & 296,740 & 0,652 & 0,952 \\
\hline 9A- Se desvaloriza mi trabajo y nunca se me reconoce que algo lo haya hecho bien & 20,66 & 291,610 & 0,726 & 0,951 \\
\hline 10A- Se ignoran mis éxitos laborales y se los atribuyen a la suerte, al azar, 0 a otra situación externa & 20,74 & 290,991 & 0,755 & 0,951 \\
\hline $11 \mathrm{~A}$ - Se castigan mis errores o pequeñas fallas duramente & 21,04 & 296,989 & 0,678 & 0,952 \\
\hline $\begin{array}{l}\text { 12A- Se me obstaculizan las posibilidades de comunicarme con mis compañeros/as o con personas de } \\
\text { otras áreas de la empresa }\end{array}$ & 21,28 & 297,347 & 0,676 & 0,952 \\
\hline $13 \mathrm{~A}$ - Se me interrumpe continuamente cuando trato de hablar & 21,17 & 296,927 & 0,725 & 0,951 \\
\hline 14A- Se me impide expresarme & 21,34 & 296,268 & 0,750 & 0,951 \\
\hline 15A- Se me ataca verbalmente criticando los trabajos que realizo con gritos, insultos o groserias & 21,53 & 302,687 & 0,660 & 0,952 \\
\hline $\begin{array}{l}\text { 16A- Se me evita o rechaza en el trabajo (evitando el contacto visual, mediante gestos de } \\
\text { rechazo o menosprecio) }\end{array}$ & 21,33 & 298,308 & 0,737 & 0,951 \\
\hline $\begin{array}{l}\text { 17A- Se ignora mi presencia, por ejemplo, dirigiéndose exclusivamente a terceros } \\
\text { (como si no me vieran, no existiera, "ley del hielo") }\end{array}$ & 21,29 & 296,416 & 0,745 & 0,951 \\
\hline $18 \mathrm{~A}-\mathrm{Se}$ me asignan nuevas tareas sin parar & 20,80 & 296,303 & 0,634 & 0,952 \\
\hline $\begin{array}{l}\text { 19A- Me encargan trabajos que requieren experiencias o competencias superiores a las que poseo } \\
\text { con la intensión de desacreditarme }\end{array}$ & 21,39 & 298,946 & 0,710 & 0,951 \\
\hline 20A-Se me critica de tal manera en mi trabajo que ya dudo de mi capacidad para hacer bien mis tareas & 21,35 & 296,103 & 0,751 & 0,951 \\
\hline 21A- Se me bloquean o limitan las oportunidades de capacitación o formación & 20,99 & 292,871 & 0,679 & 0,952 \\
\hline 22A- Se me bloquean o impiden las oportunidades que tengo de algún ascenso o mejora en el trabajo & 20,85 & 290,444 & 0,695 & 0,952 \\
\hline
\end{tabular}


Tabla 7. Correlación item - escala total (intrumento modificado, 25 ítems).

\begin{tabular}{|c|c|c|c|c|}
\hline & $\begin{array}{l}\text { Media de la Escala } \\
\text { si el item se } \\
\text { elimina }\end{array}$ & $\begin{array}{c}\text { Varianza de } \\
\text { escala si el } \\
\text { ítem se elimina }\end{array}$ & $\begin{array}{l}\text { İtem } \\
\text { correlacionado- } \\
\text { correlación total }\end{array}$ & $\begin{array}{l}\text { Alfa de Cronbach } \\
\text { si el item } \\
\text { se elimina }\end{array}$ \\
\hline 1A- Recibo ataques a mi reputación o a mi imagen personal & 23,98 & 351,107 & 0,635 & 0,950 \\
\hline 3A- Recibo burlas, calumnias o difamaciones públicas & 24,16 & 350,282 & 0,663 & 0,949 \\
\hline 4A- Se me asignan trabajos o proyectos con plazos tan cortos que son imposibles de cumplir & 23,68 & 350,364 & 0,575 & 0,950 \\
\hline 5A-Se me ignora o excluye de las reuniones de trabajo o en la toma de decisiones & 23,77 & 349,508 & 0,572 & 0,950 \\
\hline 8A- Se extienden por mi lugar de trabajo rumores malintencionados sobre mi persona & 23,84 & 348,006 & 0,648 & 0,949 \\
\hline 9A- Se desvaloriza mi trabajo y nunca se me reconoce que algo lo haya hecho bien & 23,51 & 342,676 & 0,717 & 0,949 \\
\hline 10A- Se ignoran mis éxitos laborales y se los atribuyen a la suerte, al azar, o a otra situación externa & 23,59 & 341,991 & 0,744 & 0,948 \\
\hline $11 \mathrm{~A}-\mathrm{Se}$ castigan mis errores o pequeñas fallas duramente & 23,90 & 347,693 & 0,689 & 0,949 \\
\hline 15A-Se me ataca verbalmente criticando los trabajos que realizo con gritos, insultos o groserias & 24,38 & 354,328 & 0,656 & 0,949 \\
\hline 16A-Se me evita o rechaza en el trabajo (evitando el contacto visual, mediante gestos de rechazo o menosprecio) & 24,19 & 349,792 & 0,735 & 0,949 \\
\hline $\begin{array}{l}\text { 17A- Se ignora mi presencia, por ejemplo, dirigiéndose exclusivamente a terceros } \\
\text { (como si no me vieran, no existiera, "ley del hielo") }\end{array}$ & 24,14 & 347,742 & 0,737 & 0,948 \\
\hline 18A- Se me asignan nuevas tareas sin parar & 23,64 & 347,181 & 0,637 & 0,950 \\
\hline $\begin{array}{l}\text { 19A- Me encargan trabajos que requieren experiencias o competencias superiores a las que poseo con } \\
\text { la intensión de desacreditarme }\end{array}$ & 24,24 & 349,986 & 0,713 & 0,949 \\
\hline 20A-Se me critica de tal manera en mi trabajo que ya dudo de mi capacidad para hacer bien mis tareas & 24,20 & 346,984 & 0,753 & 0,948 \\
\hline 21A- Se me bloquean o limitan las oportunidades de capacitación o formación & 23,84 & 344,051 & 0,668 & 0,949 \\
\hline 22A- Se me bloquean o impiden las oportunidades que tengo de algún ascenso o mejora en el trabajo & 23,70 & 341,307 & 0,687 & 0,949 \\
\hline $\begin{array}{l}\text { 23A- He sido agredido(a) verbalmente por personas ajenas a mi empresa u organización, como clientes, } \\
\text { apoderados, pacientes, estudiantes, etc, }\end{array}$ & 23,65 & 356,033 & 0,441 & 0,952 \\
\hline
\end{tabular}

utilice la versión original del IVAT-Pando (de 22 ítems) o la versión modificada (25 items). Los valores se presentan en la Tabla 10.

\section{Resultados de las dimensiones del instrumento:}

\section{Presencia de Violencia}

Según los resultados del instrumento original de 22 ítems, un $76,5 \%$ de la muestra presenta un alto nivel de incidencia, un $16,6 \%$ presenta un nivel medio de incidencia y el 6,8\% reportó no haber sido objeto de algún tipo de conducta de violencia psicológica en el trabajo. Pese la alta incidencia de violencia registrada, la intensidad fue predominantemente baja o nula $(68,3 \%)$.

En la versión del IVAPT PANDO modificado con nuevos items, se obtienen resultados similares a los del original. En efecto, en el 75,3\% de la muestra la incidencia de la violencia es alta, en el $19,8 \%$ es de nivel medio) y el 4,9\% reportó no haber sido objeto de violencia psicológica en el trabajo, siendo igualmente baja la intensidad de la misma.

$\mathrm{Al}$ analizar la presencia e intensidad de la violencia psicológica por sexo, se constató que mujeres y hombres reportan en proporciones muy similares presencia de violencia laboral, y que una mayor proporción de hombres están expuestos a violencia de alta intensidad. Comparando ambas versiones del instrumento, encontramos que las diferencias entre el IVAPT PANDO original y el modificado no son significativas.
Tabla 8.

Correlaciones instrumento original - GHQ-12.

$\begin{array}{llcccc} & & \begin{array}{c}\text { Intensidad } \\ \text { Violencia }\end{array} & \begin{array}{c}\text { Presencia } \\ \text { Violencia }\end{array} & \begin{array}{c}\text { Presencia } \\ \text { Acoso }\end{array} & \text { GHQ-12 } \\ \text { Intensidad } & \text { Pearson Correlation } & 1 & 0,894^{* *} & 0,595^{* *} & 0,414^{* *} \\ \text { de la Violencia } & \text { Sig, (2-tailed) } & & 0,000 & 0,000 & 0,000 \\ & N & 614 & 614 & 434 & 573 \\ \text { Presencia } & \text { Pearson Correlation } & 0,894^{* *} & 1 & 0,402^{* *} & 0,383^{* *} \\ \text { de Violencia } & \text { Sig, (2-tailed) } & 0,000 & & 0,000 & 0,000 \\ & \text { N } & 614 & 614 & 434 & 573 \\ \text { Presencia } & \text { Pearson Correlation } & 0,595^{* *} & 0,402^{* *} & 1 & 0,310^{* *} \\ \text { de Acoso } & \text { Sig, (2-tailed) } & 0,000 & 0,000 & & 0,000 \\ & \text { N } & 434 & 434 & 449 & 423 \\ & \text { Pearson Correlation } & 0,414^{* *} & 0,383^{* *} & 0,310^{* *} & 1 \\ \text { GHQ-12 } & \text { Sig, (2-tailed) } & 0,000 & 0,000 & 0,000 & \\ & N & 573 & 573 & 423 & 641\end{array}$

** la correlación es significativa al nivel 0,01 (de 2 colas).

Presencia de acoso psicológico

En lo que se refiere a acoso psicológico, los resultados del IVAPT PANDO original de 22 items muestran que sólo el 13,9\% de las personas encuestadas reportó haber experimentado algún grado de acoso psicológico: 6,5\% en el nivel medio y 7,1\% en el nivel alto, siendo similares los resultados obtenidos del instrumento modificado. La comparación entre la versión original de 22 ítems 
Tabla 9.

Correlaciones instrumento modificado - GHQ-12.

$\begin{array}{llcccc} & & \begin{array}{c}\text { IVAPT 2 } \\ \text { Intensidad } \\ \text { Violencia }\end{array} & \begin{array}{c}\text { IVAPT 2 } \\ \text { Presencia } \\ \text { Violencia }\end{array} & \begin{array}{c}\text { IVAPT 2 } \\ \text { Presencia } \\ \text { Acoso }\end{array} & \text { GHQ-12 } \\ \text { IVAPT 2 } & \text { Correlación Pearson } & 1 & 0,892^{* *} & 0,572^{* *} & 0,431^{* *} \\ \text { Intensidad } & \text { Sig, (de 2 colas) } & & 0,000 & 0,000 & 0,000 \\ \text { de Violencia } & \text { N } & 607 & 607 & 423 & 568 \\ \text { IVAPT 2 } & \text { Correlación Pearson } & 0,892^{* *} & 1 & 0,370^{* *} & 0,384^{* *} \\ \text { Presencia } & \text { Sig, (de 2 colas) } & 0,000 & & 0,000 & 0,000 \\ \text { de Violencia } & \mathrm{N} & 607 & 607 & 423 & 568 \\ \text { IVAPT 2 } & \text { Correlación Pearson } & 0,572^{* *} & 0,370^{* *} & 1 & 0,317^{* *} \\ \text { Presencia } & \text { Sig, (de 2 colas) } & 0,000 & 0,000 & & 0,000 \\ \text { de Acoso } & \mathrm{N} & 423 & 423 & 438 & 414 \\ & \text { Correlación Pearson } & 0,431^{* *} & 0,384^{* *} & 0,317^{* *} & 1 \\ \text { GHO-12 } & \text { Sig, (de 2 colas) } & 0,000 & 0,000 & 0,000 & \\ & \mathrm{~N} & 568 & 568 & 414 & 641\end{array}$

** la correlación es significativa al nivel 0,01 (de 2 colas).

Tabla 10.

Puntajes referenciales de las escalas IVAT -Pando original y modificado.

\begin{tabular}{|c|c|c|c|c|c|c|}
\hline & \multicolumn{3}{|c|}{ IVAT - Pando 22 ítemsI } & \multicolumn{3}{|c|}{ VAT - Pando 25 ítems } \\
\hline & Alta & Media & $\begin{array}{l}\text { Baja } \\
\text { o Nula }\end{array}$ & Alta & Media & $\begin{array}{l}\text { Baja } \\
\text { o Nula }\end{array}$ \\
\hline $\begin{array}{l}\text { Intensidad de } \\
\text { la violencia }\end{array}$ & $\begin{array}{l}45 \\
\text { o más }\end{array}$ & $\begin{array}{l}\text { hasta } \\
44\end{array}$ & $\begin{array}{l}\text { hasta } \\
22\end{array}$ & $\begin{array}{l}51 \\
\text { o más }\end{array}$ & $\begin{array}{c}50 \\
\text { a } 26\end{array}$ & $\begin{array}{l}25 \\
\text { a } 1\end{array}$ \\
\hline $\begin{array}{l}\text { Presencia } \\
\text { de violencia }\end{array}$ & $\begin{array}{l}5 \\
0 \text { más }\end{array}$ & $\begin{array}{c}\text { hasta } \\
4\end{array}$ & 0 & $\begin{array}{l}6 \\
0 \text { más }\end{array}$ & $\begin{array}{c}5 \\
\text { a } 1\end{array}$ & 0 \\
\hline $\begin{array}{l}\text { Presencia } \\
\text { de acoso }\end{array}$ & $\begin{array}{l}8 \\
0 \text { más }\end{array}$ & $\begin{array}{c}\text { hasta } \\
7\end{array}$ & $\begin{array}{c}\text { hasta } \\
3\end{array}$ & $\begin{array}{l}9 \\
\text { o más }\end{array}$ & $\begin{array}{c}8 \\
\text { a } 4\end{array}$ & 3 a 0 \\
\hline
\end{tabular}

y la modificada de 25 items pone en evidencia que no hay diferencias significativas entre ambas.

Es importante observar que los resultados obtenidos en esta aplicación del instrumento a una muestra chilena son consistentes con los obtenidos en otros estudios realizados en América Latina en cuanto a que la percepción de presencia de violencia en el trabajo resulta siempre mayor que el reporte de acoso psicológico. (Tabla 11).

Finalmente, en lo que respecta a violencia ejercida por actores externos introducida en este estudio del IVAPT-Pando, los datos muestran que un 4,5\% de hombres y mujeres declaran haber sido agredido físicamente, en forma frecuente o muy frecuente por personas ajenas a la empresa o institución. Estos porcentajes se elevan cuando los y las encuestadas hacen referencia a haber sido víctimas de agresión verbal (17,4\%). Finalmente, el 11,3\% de los y las trabajadoras señalan haber sido objeto de maltrato, humillación, ridiculización o menosprecio por parte de alguna persona ajena a su trabajo. En cada uno de estos reactivos el sector comercio lidera los porcentajes.

\section{DISCUSIÓN Y CONCLUSIONES}

Los resultados de la validación del inventario mostraron que este instrumento es útil para la medición de la violencia y acoso psicológico en el trabajo en la población trabajadora en Chile, en los sectores productivos estudiados. Esta validación se realizó con la muestra más grande que ha tenido este instrumento hasta la fecha en Chile (700 casos), en trabajadores/as de los sectores de comercio, salud y educación.

De acuerdo a los datos, los ítems agregados relativos a violencia externa constituyen un nuevo factor que, a pesar de que no forman parte de las condiciones internas de trabajo, ni son promovidas por los compañeros o superiores, pueden formar parte de este instrumento y arrojan información relevante sobre la violencia en el trabajo.

Si bien los resultados de la validación mostraron que el instrumento es útil para la medición de la violencia y acoso psicológico en el trabajo en Chile, su aplicación presentó varias dificultades. En primer lugar, resulta difícil aplicar el cuestionario sin la presencia de una persona capacitada. Al igual que lo señalado por Macías $^{8}$ y otros autores ${ }^{12,13}$, en nuestra validación encontramos que los y las participantes tuvieron muchas dificultades para completar la columna B (comparación con los compañeros/as), pese a darse instrucciones sobre su llenado. Asimismo, en muchos casos, los encuestados/as no sabían cómo responder en la columna B cuando habían respondido "Nunca" en la columna A. Esta dificultad se tradujo en un alto porcentaje de datos perdidos en este apartado, que pueden explicarse por la mayor carga cognitiva que genera tanto responder de dos maneras diferentes a un mismo reactivo, como por la dificultad en compararse con otros/as compañeros/as de trabajo. De allí, pensamos que la sola presentación de los objetivos y la orientación general que se entrega para la autoaplicación en solitario no son suficientes resguardos para evitar esta dificultad. Por esta razón, creemos que la forma de aplicación más adecuada para superar esta dificultad

Tabla 11.

Resultados comparados de distintos estudios (\%).

\begin{tabular}{|c|c|c|c|c|c|c|c|c|c|}
\hline Autores / Tamaño Muestra & \multicolumn{3}{|c|}{ PRESENCIA VIOLENCIA } & \multicolumn{3}{|c|}{ INTENSIDAD VIOLENCIA } & \multicolumn{3}{|c|}{ PRESENCIA ACOSO } \\
\hline $\begin{array}{l}\text { Macía } \\
\text { (Chile) }{ }^{8} N=48\end{array}$ & 16,66 & 4,16 & 79,16 & 60,4 & 25,0 & 14,58 & 81,08 & 13,51 & 5,40 \\
\hline $\begin{array}{l}\text { González, Pincheira Moyano } \\
\text { (Chile) })^{9} \mathrm{~N}=150\end{array}$ & 5,3 & 20,7 & 74 & 70 & 20,7 & 4,0 & 91,3 & 6,7 & 2,0 \\
\hline $\begin{array}{l}\text { Pando, Aranda, Olivares } \\
\text { (Bolivia) }{ }^{11} \mathrm{~N}=311\end{array}$ & 5,2 & 18,0 & 76,8 & 86,8 & 13,2 & 0,0 & 96,4 & 1,9 & 1,6 \\
\hline 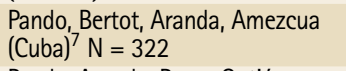 & 17,9 & 30,4 & 51,7 & 83,4 & 14,4 & 2,2 & 92,5 & 5,0 & 2,5 \\
\hline $\begin{array}{l}\text { Pando, Aranda, Parra, Gutiérrez } \\
\text { (Colombia) }{ }^{13} \mathrm{~N}=359\end{array}$ & 24,0 & 30,6 & 45,4 & 88,8 & 10,6 & 0,6 & 92,2 & 5,0 & 2,8 \\
\hline $\begin{array}{l}\text { Este estudio: Díaz, Mauro, Toro, } \\
\text { Villarroel, Campos (Chile) } \mathrm{N}=700\end{array}$ & 4,9 & 19,8 & 75,3 & 58,2 & 31,5 & 10,2 & 86,1 & 7,3 & 6,6 \\
\hline
\end{tabular}


sería la autoaplicación en presencia de una persona capacitada que pueda, no solo dar instrucciones precisas, sino responder a dudas mientras los participantes responden la encuesta.

En segundo lugar, estimamos difícil seguir la recomendación del autor del instrumento en cuanto a que este sea aplicado en el mismo lugar de trabajo y dentro de la misma jornada. Las características de las empresas e instituciones que compusieron la muestra hicieron que en la mayoría de los casos esto no pudiera cumplirse. La razón fundamental fue la negativa de los directivos de las empresas a colaborar con este estudio, rechazando dar autorización para la aplicación del instrumento. Debido a esta negativa, se aplicó el instrumento con la colaboración de dirigentes sindicales. En este caso, las limitaciones se derivan de las dificultades de los y las trabajadoras para abandonar su puesto de trabajo en horas laborales, por negativa de las empresas, por temor, o por los sistemas de trabajo en cadena. En algunos casos, los menos, se agregó la dificultad de no disponer de un lugar físico adecuado que permitiera responder la encuesta en forma grupal, ante lo cual se entregó a las personas la encuesta en sobre cerrado, previa instrucción para responderla.

\section{Agradecimientos:}

Los y las autoras agradecen la asesoría metodológica del Psicólogo Cristóbal Hernández Contreras, doctorante en Psicoterapia de la Universidad Católica de Chile.

\section{REFERENCIAS}

1. Vézina M. Les problèmes de santéreliés à l'organisation du travail: Axes de réflexion. Congrès de l'Association des médecinsspécialistes en santécommunautaire du Québec. Nov. 2000; Montreal, Canada.Montreal:AMSSCQ; 2000.

2. Hoel H, Rayner $C_{1}$ Cooper C. Workplace bullying. In: Cooper C, Robertson I.International review of industrial and organizational psychology.Chichester: Wiley; 1999. p. 195-230.

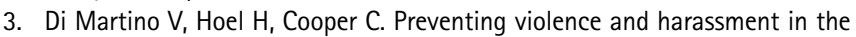
workplace. Luxembourg: European Foundation for the Improvement of Living and Working Conditions; 2003.

4. Di Martino V. Violence at the workplace. The global response. AfrNewsIOccup Health Safe.2002;12:4-7.

5. Chapell D, Vi Martino, D. La ViolenceauTravail.Geneve: BIT; 2000.

6. Pando M, Aranda B, Preciado M, Franco M, Salazar E. Validez y confiabilidad del inventario de violencia y acoso psicológico en el trabajo (Ivapt-Pando). Enseñlnvestpsicol. 2006; 11(2):319-332.

7. Pando $M$, Bertot $M$, Aranda $C$, Amezcua $M$. Análisis factorial exploratorio del inventario de violencia y acoso psicológico en el trabajo (IVAPT) para Cuba. RevMex Salud Trab. 2011;1(8).
8. Macias F. Aplicación y evaluación psicométrica del test Ivapt-Pando en dos Centros de Atención Primaria. Psicol Salud. 2008;18(002):247-254.

9. González $M$, Pincheira $P$, Moyano E. Análisis de fiabilidad y estructura factorial del inventario de violencia acoso psicológico en el trabajo IVAPT - PANDO. En:

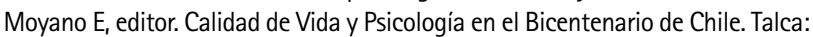
Universidad de Talca; 2010. p.507-533.

10. Matsunaga M. How to Factor-Analyze Your Data Right: Do's, Don'ts, and How-To's. Int J Psychol Res. 2015;3(1):97-110.

11. Pando $M$, Aranda $C$, Olivares $M$. Análisis factorial confirmatorio del inventario de violencia y Acoso psicológico en el trabajo (IVAPT-Pando) para Bolivia y Ecuador. Liberabit. 2012;18(1):27-36.

12. Tovalin $H$, Rodriguez $M$, Unda $S$, Centeno J, Martinez $E$, Orduña J et al. Validación y comparación de dos instrumentos para detectar la presencia de hostigamiento psicológico en el trabajo. 1er Congreso internacional y $4^{a}$ Foro de Las Américas en la Investigación sobre Factores Psicosociales, estrés y Salud Mental en el trabajo; 17-20 oct. 2012;Bogotá, Colombia. Bogotá: Universidad de los Andes; 2012.

13. Pando $M$, Aranda $C$, Parra L, Gutiérrez A. Determinación del Mobbing y Validación del Inventario de Violencia y Acoso Psicológico en el Trabajo (IVAPT) para Colombia. Salud, Barranquilla. 2013; 29(3):525-533 